\title{
Optimization Problems in Wireless Sensor Networks
}

\author{
Ada Gogu and Dritan Nace \\ Laboratoire Heudiasyc UMR CNRS 6599 \\ Université de Technologie de Compiègne \\ 60205 Compiègne Cedex, France \\ \{ada.gogu,dnace\}@hds.utc.fr
}

\author{
Arta Dilo and Nirvana Meratnia \\ Pervasive Systems group, EWI \\ University of Twente \\ 7500 AE Enschede, The Netherlands \\ \{a.dilo,n.meratnia\}@ewi.utwente.nl
}

\begin{abstract}
The Wireless Sensor Networks (WSNs) design related questions give rise to new complex and difficult theoretical problems and challenges in operations research and optimization areas. As WSNs become increasingly pervasive, a good understanding of these problems in terms of theoretical complexity is of great help in designing appropriate algorithms. In this paper, we examine some of the most fundamental optimization problems related to coverage, topology control, scheduling, routing and mobility in WSNs. Then we focus on their complexity and analyze the differences that exist with the counter part conventional theoritical problems or those already studied in traditional networks. We present as well some of the main methods proposed in the literature and report some open issues regarding these problems.
\end{abstract}

Keywords-WSN, Optimization problems, Complexity.

\section{INTRODUCTION}

WSNs present an interesting field of research due to their several applications and their integration toward more complex network systems. The difficulties in WSNs are usually related to their stringent constraints such as energy, bandwidth and memory. In this study, we investigate some fundamental problems related to coverage, routing and sink mobility, modeled as standalone optimization problems or integrated in protocols design. We notice that the proposed solution methods come from various fields of research including computational geometry; linear, nonlinear and constraint programing; metaheuristics and approximated methods, etc. However our main goal is to firstly identify these problems from the broad range of works related with the above topics. Further, we intend to make the connection with the conventional optimization problems. Finally, we report some similar problems encountered in traditional networks and discuss the differences between them. Generally speaking there are two large groups of works in WSN field, those classified as application oriented with simulation, comparative and/or real hardware studies, and those falling in the theoretical oriented studies. We consider both types of work but put the focus on the latter one. The utility of theoretical studies is twofold: firstly they allow to build ideal solutions in order to measure the effectiveness of the implemented methods and the analysis of their behavior, and secondly they propose new methods complying with limited computation capacity, sensors energy, etc.
The reminder of this paper is organized as follows. In section II we present some of the main problems related to coverage, topology control, scheduling, routing and sink mobility. For each of them, we describe the respective optimization problem formulation and discuss the proposed solutions. In section III, we discuss the open issues and conclude the paper.

\section{PROBLEMS DEFINITION}

\section{A. Coverage problems}

Maximizing the coverage of the monitoring area has received a lot of attention in literature. The problem of guarantying the coverage while meeting some application requirements gives quite often raise to NP-hard optimization problems. We analyze some basic coverage problems studied in the literature:

1) Best and worst coverage paths: Coverage is often considered as a parameter of quality of service in WSNs [39]. From this perspective, coverage describes how well the sensors observe their target area. This problem becomes more difficult for moving targets. In this case, one needs to find out the path that maximizes the distance between the moving target and the nearest sensor node, called the maximal breach path. It was shown in [16] that this problem is NP-hard. The counterpart of the above is the best-case coverage. The best-case coverage means finding areas of high observability from sensor nodes and identifying the best support and guidance paths. The maximal support path between two given endpoints consists of the path which minimizes the maximal distance between every point on the path and the nearest sensor node. Hence, it represents the shortest path connecting the two endpoints which passes as close as possible to sensor nodes.

To solve the aforementioned problems, most of works propose methods relying on computational geometry and graph theory. In order to compute the maximal breach path, Meguerdichian et al. in [39] suggest constructing the Voronoi diagram for the set of nodes. The edges of Voronoi diagram provide the points of space which have the maximal distance to the given sensors set. These edges are valuated with the distance to the nearest sensor. In this graph, the maximal breach path is a path maximizing the minimal weight of its 


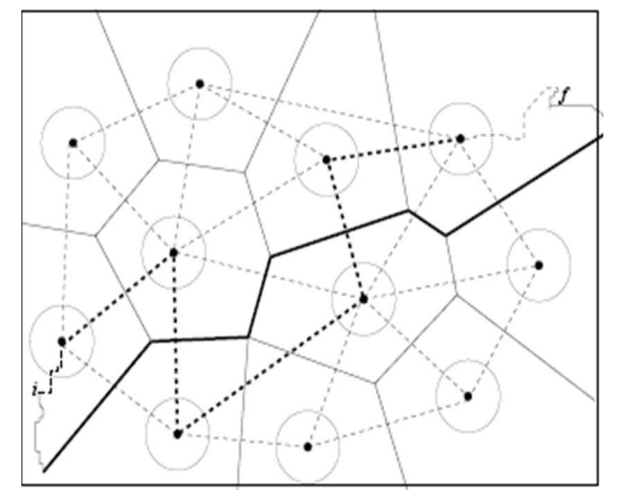

Figure 1: Maximal Breach Path (bold line) and Maximum Support Path (dashed bold line). ${ }^{1}$

edges. Breadth-first-search (BFS) algorithm is then applied to find the maximal breach path. Contrarily to the above, the maximal support path can be calculated using the Delaunay triangulation.These paths are depicted in Figure 1.

2) Detecting events and uncovered areas: In worst-case coverage, attempts are made to quantify the quality of service by finding areas of lower observability from sensor nodes and detecting breach regions. The problem, called Sensor Location Problem (SLP) formulated by [11] is stated as follows: given a planar region, one needs to position a finite number of sensor nodes so that the probability of detecting an event in this region is maximized. The non-detection probability is expressed as a function of the distance between the sensor and a given position in the space where the event may take place. The objective function is given as product of probabilities of all sensors and the problem should minimize the maximum of this product. According to this formulation, the problem is a difficult nonlinear nonconvex programming problem.

We note that a similar problem, encountered by wireless communication community is concerned with optimal deployment of base stations within a region providing the maximum possible coverage. This problem comes to be the classical weighted $p$-center location problem, where the objective is to locate $p$ identical facilities on a network that minimize the maximum of weighted distances between the clients and the corresponding (closest) facilities, assuming that each client is served by the closest facility [6]. On the other hand, the $p$-center problem is slightly different from the above SLP ${ }^{2}$. In principle, p-center makes an assignment because each demand is assigned to a facility while in SLP the event point (demand) can be captured by many sensor nodes (facilities) and hence, the objective function of SLP involves the product of probabilities of not detecting an

\footnotetext{
${ }^{1}$ The figure is taken from [16].

${ }^{2}$ For the SLP problem, the clients are presented by the events and the facilities by the sensors
}

event. Another similar problem is encountered in content distribution networks (CDNs). Streaming services such as Video on Demand (VoD) or digital television over the Internet (IPTV) are very bandwidth-intensive and caching popular streams at proxies is a potential solution. The corresponding problem is as well a $p$-center problem.

3) Optimal sensor deployment: The WSN deployment (or layout) problem is concerned with the minimization of the deployed sensor nodes while ensuring full coverage and connectivity of the monitoring area. As presented in [17], this problem is a variant of Art Gallery problem known as a NP-hard optimization problem. The Art Gallery problem asks for placing the smallest number of guards (the sensors in this case) such that every point of a certain geometric region can be surveyed by the guards. The Efrat et al. in [17] show that also the problem of deciding if $k$ sensors suffice to survey a region such that every point of this region is covered by three sensors is NP-hard.

Most of the proposed algorithms for the layout problem stem from the metaheuristics or approximated methods category. Hence, an approximation algorithm based on geometry calculations is proposed in [17]. Work presented in [14] uses a new algorithm called Guided Hyper-plane Evolutionary Algorithm (GHEA). GHEA behaves basically as a multiobjective evolutionary algorithm manipulating population and individuals. The novelty of GHEA is on the evaluation of the population based on the hyperplane. This hyperplane consists of points which have better performance than any individual of the current population. Work presented in [19] proposes an ant colony algorithm for this problem. Such algorithm follows the behavior of real ant colony where the more the ants follow a trail, the more attractive the trail is. The ants lay down quantities of pheromone (chemical cues) to mark the path they follow. Similarly, the ant algorithm calculates a pheromone value for every step of the iteration and a local estimated value. Then, the forwarding decision for each ant is a probabilistic one calculated over these values. In order to apply the ant algorithm for the layout problem, the area is modeled as a grid and the pheromone is calculated for each point of this grid, while the local information includes such information as the number of covered points for every location, connectivity between two points, etc.

\section{B. Topology control}

The primary concern of the topology control problems is to ensure the connectivity of the network. According to the application, some other criteria such as minimizing the energy consumption, k-connectivity, can be considered for these problems. Some of the most studied topology control problems follow.

1) Backbone-based topology: With respect to a hierarchical network architecture, the backbone-based topology 
corresponds to a subset of nodes that guarantee the connectivity of the whole network. Every node excluded from this set must have at least an edge in common with a node in the backbone subset. This problem is modeled as a widely known mathematical problem called the Connected Dominating Set (CDS). A Dominating Set (DS) of a graph $G\left(V_{\text {nodes }}, E_{\text {edges }}\right)$ is the subset of nodes $D \subset V$, such that every node that does not belong to $D$ has at least one link in common with a node in $D$. In the special case where these nodes have to be connected, the problem is called Connected Dominating Set (CDS). For many applications, the smallest dominating set is desired. This brings us to the problem of finding the Minimum Connected Dominating Set (MCDS). MCDS is shown to be a NP-hard optimization problem. The nodes in a CDS are called dominators, the others are called dominatees. This problem is of the same difficulty and directly convertible to the vertex cover, independent set computation problem, or the maximum clique one. [57] proposes a method to obtain the CDS which is basically built in two phases: During the first phase, a Maximal Independent Set (MIS) ${ }^{3}$ is formed. In the second phase, the goal is to build a $C D S$ using nodes that do not belong to the MIS. These nodes are selected in a greedy manner. At the end, the non-MIS node with the highest weight (the weight depends on the remained energy and the node degree) becomes part of the $C D S$. Unfortunately, a $C D S$ only preserves 1-connectivity and it is therefore very vulnerable. When taking in account the fault tolerance due to node failures, the problem is known as the kmCDS problem, (k-Connected m-Dominating Set). The requirement of kconnectivity guarantees that between any pair of dominators there exist at least $k$ different paths and the m-domination takes care of fault tolerance and robustness for dominatees. $\mathrm{Wu}$ and $\mathrm{Li}$ in [55] propose a distributed algorithm for this problem with time complexity $O((m+\Delta) \cdot$ Diam $)$, where $\Delta$ is the maximum node degree and Diam is the diameter ${ }^{4}$ of the network. The problem becomes much more complicated for dynamic or mobile networks.

2) Strong minimum energy topology: Given a set of sensor nodes scattered in an area, the problem of strong minimum energy topology (SMET) is to find the node's transmission power such that the energy consumption for all nodes is minimized while preserving at least a bidirectional connectivity between two pairs of nodes in the network. Notice that the power of each node determines the network connectivity and hence the topology. Some related works refer to this problem as the Range Assignment (RA) problem.

SMET is proved by Cheng et al. in [12] to be a NPHard optimization problem. To tackle the problem they

\footnotetext{
${ }^{3}$ An Independent Set $(I S)$ of graph $G$ is the node subset $S$ where no two nodes in $S$ have an edge in common. The $M I S$ is the maximal $I S$, which means that it is not possible to include more nodes in $S$.

${ }^{4}$ The diameter of a given connected graph is the length of the longest shortest path between any pair of nodes in the graph.
}

propose two heuristics: the Minimum Spanning Tree (MST) where the power is assigned to nodes such that they can reach the farthest neighbor in the MST and the Incremental Power. Since transmitting with the same power can lead to energy waste, some methods based on computational geometry such as Relative Neighbor Graph [37], Gabriel Graph [31] or Voronoi Diagram and Delaunay Triangulation were introduced to determine the "best neighborhood". These methods intend to assign to every node a given power such that it can reach its one-hop "neighbors". If a node realizes that the energy cost to directly transmit to some nodes would be smaller than the cost of using any other relaying scheme to reach them, then it will call these nodes as its own "neighbors". These methods are implemented in a centralized manner but there also exist distributed variants [36]. Konstantinidis et al. in [35] propose a memetic algorithm (a combination of the genetic algorithm with the local search) called ToCMA to solve the SMET problem. In fact, the difficulty of applying this kind of algorithms stands in modeling the problem according to the algorithm's logic (e.g. how to define a chromosome, how to implement crossover, the population diversity, etc.). A ToCMA solution to the SMET problem is represented by a positive integer array where the elements of the array correspond to the power levels assigned to each node, and the respective indexes correspond to the node ID. This array is considered as individual, chromosome or solution depending on the point of view. The objective of the SMET problem is given by the fitness function defined by the sum of the power assigned to each node. The first phase of the algorithm proceeds by initializing a random population and finding feasible solutions. While the second phase applies a genetic algorithm and selects the best individual. The procedure is repeated until the solution cannot be further improved.

Another variation of this problem intends to minimize the maximum transmission power consumed at each node instead of the total energy consummation by all nodes. Work in [10] formulates this problem as an Integer Programming (IP) for a sensor network composed of sensor nodes and supernodes. They solve this problem using three approaches; an IP solver, a distributed greedy heuristic and a cluster strategy where for each cluster the nodes are organized according to a local minimum spanning tree. Another distributed protocol is presented by Costa et al. in [13] where each node reaches the $k^{t h}$ neighbor by incrementing its power gradually.

Other works propose more light heuristics, for instance [22] assumes that the node assignment power has to depend on the traffic it handles. Thus, it proposes how to compute in a greedy manner the optimal transmission range of the sensors knowing the traffic load that they have to transmit. Whereas [24] discusses the problem of joint transmission range and optimal sensor deployment to reduce the energy consumption using the dynamic programming method. It 


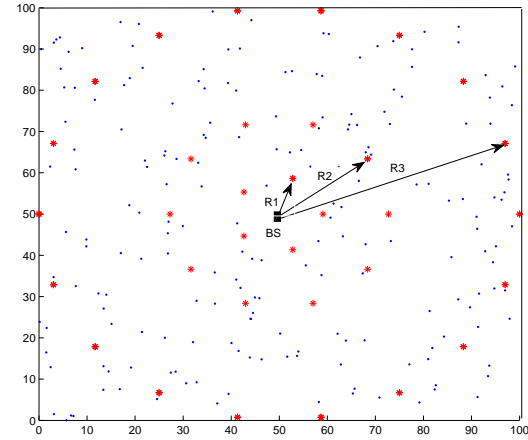

Figure 2: Joint deployment and range assignment in WSN

finds the optimal number of sensor nodes and their transmission range for a linear network according to different traffic scenarios. This work is extended for the multihop tree network case (Figure 2). In the figure the red points present the optimal configuration of positions in the network where the sensors can be placed to ensure the data transmission with minimal energy consumption.

\section{Mobility}

Data collection by mobile sinks is introduced as an alternative solution for coverage and connectivity or extending the lifetime in WSNs. Usually the problems concerned with finding the shortest path in order to reduce both sink travel cost and data collection time are modeled as the well known Travel Salesman Problem (TSP) ${ }^{5}$. For dense sensor networks [2] proposes to firstly organize the sensors in clusters and then to solve the classical TSP going through the clusters. Yuan et al. in [56] assume that sensor nodes have different transmission ranges, so the above problem is defined as Traveling Salesman Problem with Neighborhood (TSPN), which is also a NP-hard problem. In TSPN a salesman needs to deliver products to a group of people in different places and the clients can meet the salesman within a certain region near their house. Whereas, the problem of moving the sensors to ensure the coverage discussed by [27] is based in potential field approach. The authors make the nodes subject of some virtual forces and apply the physical laws. Other works like [40] achieve the actuator's energy saving by limiting the broadcast transmission and reducing the number of positions updates for a given network with static sensors and mobile actuators. Indeed, the broadcast transmission area is limited to Voronoi cell and the actuators positions will be triggered only when the sensor's prediction is bigger than a threshold. The sensors use the Kalman filter to predict the actuator's movement. While in [4] the

\footnotetext{
${ }^{5}$ Given a number of cities and their respective distances, TSP consists in finding the shortest path that visits each city exactly once.
}

information storing process for nodes mobility is modeled through autoregressive (AR) estimation models.

\section{Scheduling}

Many works in WSN domain are concerned with scheduling problems. They are basically intended to bring solutions for the interference and collisions avoidance, for the purpose of channel contention management, but also for lifetime maximization or QoS awareness goals.

1) Scheduling based on TDMA scheme : TDMA (Time Division Multiple Access) permits the nodes to access the medium by assigning a time slot to each of them. The scheduling problem is concerned with computing a time slot allocation for every node in the network such that two neighboring nodes do not transmit at the same time slot. The TDMA accessing scheme is employed quite often to ensure a collision-free MAC but its implementation in WSNs, mainly due to synchronization requirements, is not easy. Most of these problems are shown to be NP-hard and are mathematically modeled as vertex or edge graph coloring problems.

In several works, for instance [21] and [30], the time slot allocation problem is formulated as a graph coloring problem. Gandham et al. in [21] propose a greedy based heuristic for the edge coloring problem where two edges incident on the same node can not be allocated at the same time slot. In a first round, they implement a greedy heuristic to color the edges while in the second round they map the colors to the time slots. Kawano et Miyazaki in [30] propose a distributed algorithm where each node has to know the list of its two-hop reachable nodes, their color, and the number of hops from the nearest base station. It also has to keep a variable updated which indicates how many packet collisions occur at a time. They evaluate their algorithms by using as criteria the number of packet collisions in the network. This slot allocation mechanism is also encountered in [3], [30], [50]. In contrast with the others, the work in [3] assumes different frequencies for transmission and receiving which results in reducing the number of time slots in the frame. The authors describe also the construction of an energyaware data gathering tree to ensure that all data are delivered to the sink. The data transmission phase is repeated many times for the same schedule in the same round.

Another version of this problem looks for the chromatic number, that is the minimal number of colors necessary to color a graph. Hence, the objective is to find a minimumlength interference-free schedule that satisfies the data transmission requirements (e.g. the flow rates, the delays) for all the nodes. Kedad et al. [32] formulate the problem as follows: how to construct a frame with the minimum number of time slots such that the activated links a) are not in conflict for each time slot and b) creates a strongly connected graph. They show that this problem is NP-hard and propose two approximated algorithms. In [18] the problem is to find a 
minimum length frame during which the nodes can deliver all their data to the sink. They reduce this problem to finding the chromatic number of a graph. The difference with the aforementioned works stands in considering the interference graph $^{6}$ and scheduling the edges more than once in a time frame. The work of [53] transforms the problem in a multi-objective optimization problem. The first objective is to minimize the time delay of data delivery and the second one is to minimize the energy while taking not only the transmission and receiving energy into account but also the energy consumed for transition between sleep and active mode. In fact, these objectives contradict each other as the energy objective intends to maximize the number of nodes turned off, which in turn increases the delay. The optimization problem intends to solve the trade-off between energy and delay by using the particle swarm optimization approach proposed by [33].

2) Scheduling and coverage: One of the most interesting application of scheduling theory is determining the duty cycle of nodes which aims at putting the nodes in sleep periodically in order to minimize the power consumed during idle listening. Let's see how the duty cycle concept is applied to a redundant sensor network with a set of targets to be monitored. The problem is how to group the nodes such that each group (called cover) is able to cover the targets while maximizing the WSN lifetime. To save energy the nodes belonging to a cover will be switched off for a period of time if another cover set for the same area will be active. Thus, the covers will be activated in a sequential manner. This problem may require also to guarantee a given number of nodes for every cover set to ensure a given fault tolerance threshold. This problem is shown to be NP-hard in [15]. In [2] it is modeled as the weighted set cover problem (SCP). The problem assumes that it exists a dense sensor network and a set of demand points $D$ which represent the center of a small area in the deployment field. Each node can monitor a subset of the demand points only if they are inside the sensor node's sensing range. On the other side, the demand points have to be covered by at least one sensor node. The activation cost of every node is proportional to the inverse of its remaining energy. Then the problem is to find a set of nodes such that all demands are covered by at least one node and the sum of activation energy is minimized. The calculations are done off-line and then injected to the sink. When the mobile sink gathers data from the nodes it also indicates to them whether they will be active in the next period. In [47], the problem is modeled as a linear program which intends to maximize the sum of covers lifetime and the constraint is that the sum of the node activation time is less than the lifetime of its own battery. A distributed solution is proposed in [23], in which wake-up times of

\footnotetext{
${ }^{6}$ An interference graph $C=(V, I)$ is constructed such that, if either edge $u$ or $v \in I$ can hear each other, then $(u, v) \in I$.
}

nodes and wake-up scattering decisions are based on random assignments. In every round (its length is assumed given), the wake-up scattering defines the waking-up time of the nodes. In terms of coverage, it intends to spread the wakeup interval of nodes in the network as evenly as possible in order to maximize the event detection probability for a given area. Indeed, the algorithm does not take into account that the targets are continuously monitored. Both [45] and [15] propose a distributed solution for coverage and include in their study the problem of dependence between the covering sets.

\section{E. Routing}

The problems of data transmission in WSNs through energy-efficient flow paths, known as routing problem, can be formulated as classical graph flow problems. These problems can be classified as minimum cost flow problems, multicommodity flow problems, shortest path or minimum spanning tree problem. While many works related to flow problems have been done for wired networks, some new challenges have appeared for wireless networks and especially for WSN. In the network flow problem formulation, the link capacity is a strong constraint, which is substituted by the node energy constraint in the WSNs. Also for wireless networks, one needs to add in the model the interference caused by broadcast nature of wireless links. However the main differences between these two paradigms stand on including the dynamic topology models and implementing distributed solutions for wireless networks.

In earlier works, the flow problems are formulated as shortest path tree (SPT) or spanning tree (ST) problem as in [46] and [44]. The Minimum Total Energy (MTE) routing algorithm [46] finds the path with the minimum total energy consumption while Minimum Hop (MH) [44] algorithm uses the number of hops as a metric to achieve energy efficient routing. These algorithms are time polynomial and mainly based on Dijkstra's or Bellman Ford algorithms. The tree construction based on a spanning tree which minimizes the total sum of edge costs (Minimum Spanning Tree) is also suggested as a structure for data gathering. The problem is polynomial and some frequently used algorithms are Prims or Kruskal [5]. But minimizing the total energy consumption is not enough since some nodes deplete their energy faster than others and may cause network partition. To handle this, an approach is to minimize the maximum energy consumption of the nodes. This problem has been modeled by [20] as the minimum degree spanning tree (MDST), a NP-hard optimization problem.

In addition to SPT and MST, the routing problem is modeled as an optimization flow problem aiming at energy minimization or lifetime maximization in multi-commodity WSNs. Sankar and Liu in [49] address the problem of lifetime maximization while accommodating the generated data by multiple sources to the sink. Thus, the commodi- 


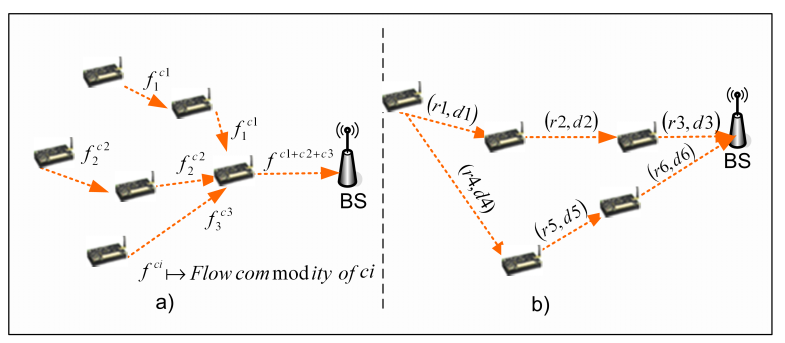

Figure 3: Illustration of a) multi-commodity flows and b) multipath routing with two metrics

ties are differentiated by their origin-destination pairs but binded together because of common link capacities sharing. The problem defines the data rates of every link for each commodity in order to maximize the lifetime until the first node fails. It is shown in [29] that the multi-commodity flow problem is NP-hard in a directed graph even for two commodities. Figure 3 illustrates the concept of the multicommodity flows in a graph and the QoS multipath routing with two metrics.

As expected, routing problems involving QoS requirements become much more difficult to handle. A first approach to guarantee QoS requirements in routing is to consider edge cost as a combination of several metrics such as number of hops, residual energy or buffer size, while the problem itself aims at finding a number of disjoint paths such that the cost of each path is lower than a given threshold cost, [38]. But in this case, a combined metric does not guarantee that the applications' requirements are met. To illustrate this, let's look at [7] where the QoS routing problem is concerned with delay and reliability criteria. The goal is to find the smallest set of disjoint paths between a source and a destination such that both criteria are satisfied and the energy consumption is minimized. Delay presents a stringent metric, meaning that if the delay is not respected in any of the set paths, the packet is dropped. While, the reliability of every connection source-destination follows the multiplicative composition rule, hence the more path we add in the set, the more reliable the set will be. The problems of finding the path which minimizes the energy or the delay, or maximizes the reliability, taken separately are solvable in polynomial time, while the whole problem is not. Indeed, constructing a path that satisfies the constraint of reliability and delay is shown to be NP-hard, see [54]. Finally the general problem asks to find the smallest cardinal of the paths set which minimizes the energy and its disjoint paths satisfy both the constraints. As the problem for a single path is NP-hard, it follows that for any number $N$ of paths the general problem remains NP-hard.

Flow problems are quite often formulated as a linear program (LP) [9], [26], [42]; max-min LP [51], integer LP [1], [28], mixed integer LP [25], and also as nonlinear convex (or non-convex) programming [52] and constrained optimization problem [59]. As a recent trend in WSN routing we would emphasize the utilization of metaheuristics [43], [48]. The algorithm proposed in [48] is based on ant colony optimization (ACO), a multi-agent approach. Another algorithm for routing combines ACO approach with a tabu list [43].

The new paradigm of opportunistic routing (OR) [58], [59] has been developed in WSN routing thanks to its property for overcoming the problems of unreliable links or unstable end-to-end connections. OR makes use simultaneously of the network and MAC layers. The network layer selects the best candidate nodes to forward a packet to and at the MAC layer one node is selected dynamically as the actual forwarder. The decisions in routing and scheduling are taken hop-by-hop opportunistically. Niu et Cui [41] study simultaneously the routing and scheduling in OR. They state that this problem is NP-hard. In [34], a QoS routing scheme is studied, that is how to route the data efficiently and guarantee the time requirements. As depicted above, the problem is NP-hard and [34] proposes an heuristic according to the opportunistic approach. Note that the OR would be very exhausting in energy for traditional networks because of multiple broadcasts needs.

\section{OPEN ISSUES}

Several issues in WSNs are still open or not sufficiently addressed. In particular we believe that uncertainty is an important characteristic of WSNs which has been very little taken into account until now. The uncertainty is due to the nature of WSNs and relates to situations such as data delivery, event detecting etc. Some attempts to model these situations use probabilities associated with such events. The difficulty of taking the uncertainty of WSNs into account is mainly related to two factors. Firstly, measuring the distribution of events is not an easy task and is both environment and application dependent. Secondly, despite recent advances on robust optimization ${ }^{7}$ tackling such optimization probabilistic problems is difficult. Other issues that in our point of view have received less attention are scalability, the discontinuous nature of solutions or considering mobility in the optimization problems. To illustrate this let us consider the RA problem. The range transmission assignment problem should further emphasize two aspects, the first one concerns the fact that the power transmission takes a finite number of values while concerns a large number of possibly different types of sensors, and the second one expresses the relation between the transmission distance and the power on the one hand and the transmission distance and the success probability of a message sent on this link which impacts the energy on the other hand. The scalability comes also together with multi sinks, and the need/problems

\footnotetext{
${ }^{7}$ Indeed, since the works of Bertsimas and Sim [8], who have shown how to model an optimization stochastic problem as a Linear Program under weak conditions, robust optimization has been intensively investigated.
} 
for multi-sink/multi-commodity WSN design, which are not sufficiently considered in the theoretical studies.

With respect to the coverage problems, there are several potential directions that have not been fully explored, i.e.: solving the deployment problem in the presence of the obstacles, taking into account the restrictions for node placement, or still extending the work for $3 D$ deployment. For routing and topology control scalability, links' reliability and multiple mobile sinks remain challenging.

Another crucial issue is the difference that still exists between the practical implementation and theoretical studies. In this aspect there is still an important gap to fill. Most of the works presented in section II are centralized and off-line computation, while protocols implemented in real hardware or tested in simulations are on-line and distributed. All this needs sensors being more collaborative, meaning considering neighborhood for decisions not just one sensor node. Fusing the two is not straightforward, there is work to be done in that direction. Another difference is that optimization problems assume/work with a static environment, meaning no dynamic network, no dying nodes, even less node mobility. Dynamicity is a main consideration in WSN protocols but however including it in optimization problems models is still a challenge. Last, optimization studies considering cross-layer, e.g. scheduling combined with routing, are not many because it increases complexity despite the practical interest of such studies.

\section{REFERENCES}

[1] A.B. Aguiar, A.Neto, P.R. Pinheiro, and A. L.V. Ceoelho. Applicability of a novel integer programming model for wireless sensor network. IJCSIS, 3, 2009.

[2] W. Aioffi, G. Mateus, and F. Quintao. Optimization issues and algorithms for WSNs with mobile sink. In: Proc. INOC, 2007.

[3] T.A. Al-Khdour and U. Baroudi. An energy-efficient distributed schedule-based communication protocol for periodic wireless sensor networks. Arabian journal for science and engineering, 35:155-168, 2010.

[4] M. Ali, T. Voigt, and Z.A Uzmi. Mobility management in sensor networks. DCOSS, pages 131-140, 2006.

[5] S. A. Attarde, L. L. Ragha, and S. K. Dhamal. An enhanced spanning tree topology for wireless sensor networks. Int. Journal of Comp App., 1:46-51, 2010.

[6] I. Averbakh and O. Berman. Minimax regretp-center location on a network with demand uncertainty. Elsevier Science, 5:247-254, 1997.

[7] A. B. Bagula and K. G. Mazandu. Energy constrained multipath routing in wireless sensor networks. UIC, pages 453-467, 2008.

[8] D. Bertsimas and M. Sim. The price of robustness. Operations Research, 1:35-53, 2004.
[9] M. Borghini, F. Coumo, and F. Ricciato. Optimal data delivery in wireless sensor network in the energy and latency domain. WICON, pages $138-145,2005$.

[10] M. Cardei, M. Pervaiz, and I. Cardei. Energy-efficient range assignment in heterogeneous wireless sensor network. ICWMC, pages 11-17, 2006.

[11] T. M. Cavalier, W. Conner, E. Castillo, and S.I. Brown. A heuristic algorithm for minimax sensor location in the plane. Europian Journal of Operational Research, pages 42-55, 2007.

[12] X. Cheng, B. Narahari, R. Simha, M. X. Cheng, and D. Liu. Strong minimum energy topology in WSNs: NPCompleteness and heuristics. IEEE Trans. on Mob. Comp., 2:248 - 256, 2003.

[13] P. Costa, M. Cesana, S. Brambilla, and L. Casartelli. A cooperative approach for topology control in wireless sensor networks. Perv. and Mob. Computig, 5:526-541, 2009.

[14] C.Rotar, M. Risteiu, I. Ileana, and C. Hutanu. Optimal sensors network layout using evoutionary algorithms. Proceeding of WSEAS, pages 88-93, 2009.

[15] A. Dhawan and S. K Prasad. A distributed algorithmic framework for coverage problems in wireless sensor networks (2009). IJPEDS, pages 18-25, 2009.

[16] A. Duttagupta, A. Bishnu, and 1. Sengupta. Maximal breach in WSNs: Geometric characterization and algorithms. Algosensors, pages $126-137,2008$.

[17] A. Efrat, S. Peled, and J. Mitchel. Approximation algorithms for two optimal location problems in sensor networks. Broadband Networks, 1:714-723, 2005.

[18] S. Ergen and P. Varaiya. TDMA scheduling algorithms for WSN. Wireless Networks, 16:985 - 997, 2010.

[19] S. Fidanova, P. Marinov, and E. Alba. ACO for optimal sensor layout. Proceeding of International Conference on Evaluationary Computation, pages 5-9, 2010.

[20] A. Gagarin, S. Hussain, and L. T. Yang. Distributed search for balanced energy consumption spanning trees in wireless sensor networks. Adv. Inf. Net. and App. Work., pages 975982, 2009.

[21] S. Gandham, M. Dawande, and R. Prakash. Link scheduling in sensor networks: distributed edge coloring revisited. Infocom, 4:2492-2501, 2005.

[22] Q. Gao, K.J.Blow, D.J. Holding, I.W.Marshall, and X.H. Peng. Radio range adjustment for energy efficient wireless sensor networks. Elsevier, Ad Hoc Networks, pages 75-82, 2006.

[23] A. Giusti, A. L. Murphy, and G. P. Picco. Decentralized scattering of wake-up times in WSNs. EWSN, pages 245260, 2007.

[24] A. Gogu, D. Nace, and Y. Challal. A note on joint optimal transmission range assignment and deployment for wireless sensor networks. IEEE Networks, pages 1-6, 2010. 
[25] E. Guney, N. Aras, K. Altinel, and C. Ersoy. Efficient integer programming formulations for optimum sink location and routing in heterogeneous WSN. Computer Networks, pages 1805-1822, 2010

[26] B. Hong and V. Prasanna. Constrained flow optimization with applications to data gathering in sensor networks. Algosensors, pages 187-200, 2004.

[27] A. Howard, M. Mataric, and G. Sukhatme. Mobile sensor network deployment using potential fields: A distributed, salable solution to the area coverage problem. DARS, pages 299-308, 2002.

[28] J.N. Karaki, R. Ul-Mustafa, and A.E. Kamal. Data aggregation and routing in WSN : Optimal and heuristic algorithms. Computer networks, pages 945-960, 2009.

[29] R.M. Karp. On the complexity of combinatorial problems Networks, 5:45-68, 1975.

[30] R. Kawano and T. Miyazaki. Distributed data aggregation in multi-sink sensor networks using a graph coloring algorithm. In Proceedings of IEEE AINA, pages 906-912, 2009.

[31] W. Ke, W. Liqiang, C. Shiyu, and Q. Song. An energy-saving algorithm of WSN based on Gabriel graph. WiCom, pages $1-4,2009$

[32] S. Kedad, F. Pasqual, and P. Fouilhoux. Ordonnancement de paquets dans les réseaux sans fil. In Proc. of ROADEF, 2010.

[33] J. Kennedy and R. Eberhart. Particle swarm optimization. Proc. of IEEE Int. Conf. on Neural Netw., 4:1942 - 1948, 1995.

[34] J. Kim and B. Ravindran. Opportunistic real-time routing in multihop wireless sensor network. SAC, pages 2197-2201, 2009.

[35] A. Konstantinidis, K. Yang, H. Chen, and Q. Zhang. Energyaware topology control for WSN using memetic algorithms. Computer Communications, pages 2573-2764, 2007.

[36] X. Li, Y.Calinescu, and G. Wan. Distributed construction of a planar spanner and routing for ad hoc wireless networks. In: Proc. of IEEE Infocom, pages 1268-1277, 2002.

[37] X. Y. Li, P.J. Wan, and Y. Wang. Power efficient and sparse spanner for wireless ad hoc networks. In IEEE Int. Conf. on Comp. Com.Net., pages 564 - 567, 2001.

[38] Y. M. Luz and V. Wong. An energy-efficient multipath routing protocol for wireless sensor networks. Int. J. Commun. Syst., 20:747-766, 2007.

[39] S. Meguerdichian, F. Koushanfar, M. Potkonjak, and M. B. Srivastava. Coverage problems in wireless ad hoc sensor networks. Proc. of IEEE Infocom, 2001.

[40] T. Melodia, D. Pompili, and I. F. Akyildiz. A communication architecture for mobile wireless sensor and actor network. SECON ACM, 2005.

[41] X. Niu and L. Cui. Throughput capacity of opportunistic routing in WSN. Distributed sensor networks, 2009.
[42] T. Nivas and G. Zaruba. Upper bound of sensor network lifetime: A flow optimization approach. Procedings of the ACM GHCCWC, 2007.

[43] S. Okdem and D. Karaboga. Routing in WSN using an ant colony optimization (ACO) router chip. Sensors, pages 909921, 2009.

[44] C. Perkins and E. Royer. Ad hoc on-demand distance vector (aodv) routing. Proceeding of Work. MCSA, 1999.

[45] Sushil K. Prasad and Akshaye Dhawan. Distributed algorithms for lifetime of wireless sensor networks based on dependencies among cover sets. HiPC, pages 381-392, 2007.

[46] V. Rodoplu and T. H. Meng. Minimum energy mobile wireless networks. In Proc. IEEE ICC, , 3:1633-1639, 1998.

[47] A. Rossi, A. Singh, and M. Sevaux. Génération de colonnes dans le réseaux de capteurs sans fil. In Proc. of ROADEF, 2010.

[48] K. Saleam, N. Fisal, M. Baharudin, S. Hafizah, S. Kamilah, and R. Rashid. Colony inspired self-optimized routing protocol based on cross layer architecture for WSN. TenCon, pages $1-6,2010$

[49] A. Sankar and Z. Liu. Maximum lifetime routing in wireless ad-hoc networks. Infocom, 2:1089-1097, 2004

[50] A. Sridharan and B. Krishnamachari. Max-min fair collisionfree scheduling for wireless sensor networks. Perfor., Comp. and Communic., 585-590, 2004

[51] H. Wang, D. Peng, W. Wang, and H.Sharif. Cross layer opimization based on rate distribution in multirate WSNs. Journal of Computer Science 3, pages 368-375, 2007.

[52] Q. Wang, T. Zhang, and S. Pettersson. An effort to understand the optimal routing performance in wireless sensor network. Proc. of AINA, pages 279-286, 2008.

[53] T. Wang, Z. Wu, and J. Mao. A new method for multiobjective TDMA scheduling in WSN using pareto-based PSO and fuzzy comprehensive judgement. HPCC, 2007.

[54] Z. Wang and J. Crowcroft. Quality of service routing for supporting multimedia applications,. IEEE J. Sel. Areas Commun, 14:1228-1234, 1996.

[55] Y. Wu and Y. Li. Construction algorithms for k-connected m-dominating sets in WSN. MobiHoc, 2008.

[56] B. Yuan, M. Orlowska, and S. Sadiq. On the optimal robot routing problem in wireless sensor networks. IEEE Transactions on Knowledge and Data Engineering, 9, 2007.

[57] Z. Yuanyuan, X. Jia, and H. Yanxiang. Energy efficient distributed connected dominating sets construction in WSN. Proceeding ACM IWCMC, pages 797-802, 2006.

[58] B. Zhao and M.C. Valenti. Practical relay networks: A generalization of hybrid-ARQ. IEEE Journal of Selected Areas in Communications, , 23:7-18, 2005.

[59] J. Zhu, S. Chen, B. Bensaou, and K Hung. Tradeoff between lifetime and rate allocation problem in WSN: A cross layer approach. Infocom, pages 267-275, 2007. 